\title{
Where is my arm? Investigating the link between complex regional pain syndrome and poor localisation of the affected limb
}

\author{
Valeria Bellan $^{\text {Corresp., 1, }}{ }^{\text {, Felicity A Braithwaite }}{ }^{2}$, Erica M Wilkinson ${ }^{2}$, Tasha R Stanton $^{2}$, G. Lorimer Moseley ${ }^{2}$ \\ ${ }^{1}$ Cognitive and Systems Neuroscience Research Hub (CSN-RH), University of South Australia, Adelaide, South Australia, Australia \\ IIMPACT in Health, University of South Australia, Adelaide, South Australia, Australia \\ Corresponding Author: Valeria Bellan \\ Email address: valeria.bellan@gmail.com
}

Background. Anecdotally, people living with Complex Regional Pain Syndrome (CRPS) often report difficulties in localising their own affected limb when it is out of view. Experimental attempts to investigate this report have used explicit tasks and yielded varied results.

Methods. Here we used a limb localisation task that interrogates implicit mechanisms because we first induce a compelling illusion called the Disappearing Hand Trick (DHT). In the DHT, participants judge their hands to be close together when, in fact, they are far apart. Sixteen volunteers with unilateral upper limb CRPS (mean age 39 \pm 12 years, 4 males), 15 volunteers with non-CRPS persistent hand pain ('pain controls'; mean age 58 \pm 13 years, 2 males) and 29 pain-free volunteers ('pain-free controls'; mean age $36 \pm 19$ years, 10 males) performed a hand-localisation task after each of three conditions: the DHT illusion and two control conditions in which no illusion was performed. The conditions were repeated twice (one for each hand). We hypothesised that (1) participants with CRPS would perform worse at hand self-Iocalisation than both the control samples; (2) participants with non-CRPS persistent hand pain would perform worse than pain-free controls; (3) participants in both persistent pain groups would perform worse with their affected hand than with their unaffected hand.

Results. Our first two hypotheses were not supported. Our third hypothesis was supported - when visually and proprioceptively encoded positions of the hands were incongruent (i.e. after the DHT), relocalisation performance was worse with the affected hand than it was with the unaffected hand. The similar results in hand localisation in the control and pain groups might suggest that, when implicit processes are required, people with CRPS' ability to localise their limb is preserved. 


\section{Where is my arm? Investigating the link between complex} 2 regional pain syndrome and poor localisation of the affected $3 \operatorname{limb}$

4

5 Valeria Bellan ${ }^{1,2}$, Felicity A. Braithwaite ${ }^{2}$, Erica M. Wilkinson², Tasha R. Stanton², G. Lorimer

6 Moseley $^{2}$

7

$8{ }^{1}$ Cognitive and Systems Neuroscience Research Hub (CSN-RH), University of South Australia,

9 Adelaide, South Australia, Australia

$10{ }^{2}$ IIMPACT in Health, University of South Australia, Adelaide, South Australia, Australia

11

12 Corresponding Author:

13 Valeria Bellan ${ }^{1,2}$

14 St. Bernards Rd., Magill, SA 5072, Australia

15 Email address: valeria.bellan@gmail.com 


\section{Abstract}

19 Background. Anecdotally, people living with Complex Regional Pain Syndrome (CRPS) often report difficulties in localising their own affected limb when it is out of view. Experimental attempts to investigate this report have used explicit tasks and yielded varied results.

Methods. Here we used a limb localisation task that interrogates implicit mechanisms because we first induce a compelling illusion called the Disappearing Hand Trick (DHT). In the DHT, participants judge their hands to be close together when, in fact, they are far apart. Sixteen volunteers with unilateral upper limb CRPS (mean age 39 \pm 12 years, 4 males), 15 volunteers with non-CRPS persistent hand pain ('pain controls'; mean age 58 \pm 13 years, 2 males) and 29 painfree volunteers ('pain-free controls'; mean age 36 \pm 19 years, 10 males) performed a handlocalisation task after each of three conditions: the DHT illusion and two control conditions in which no illusion was performed. The conditions were repeated twice (one for each hand). We hypothesised that (1) participants with CRPS would perform worse at hand self-localisation than both the control samples; (2) participants with non-CRPS persistent hand pain would perform worse than pain-free controls; (3) participants in both persistent pain groups would perform worse with their affected hand than with their unaffected hand.

Results. Our first two hypotheses were not supported. Our third hypothesis was supported when visually and proprioceptively encoded positions of the hands were incongruent (i.e. after the DHT), relocalisation performance was worse with the affected hand than it was with the unaffected hand. The similar results in hand localisation in the control and pain groups might suggest that, when implicit processes are required, people with CRPS' ability to localise their limb is preserved. 


\section{Introduction}

41 People with Complex Regional Pain Syndrome (CRPS) often report severe sensorimotor

42 disturbances such as difficulty in localising their affected limb when they are not able to directly

43 look at it (Lewis, Kersten et al. 2007, Lewis, Kersten et al. 2010, Lewis and McCabe 2010). Self-

44 localisation is an important component of the self. For example, we know this body is our body

45 because: (1) it feels like it is ours (sense of ownership); (2) each body part moves how we want it

46 to move (sense of agency); (3) we know where each body part is (self-localisation) (Serino,

47 Alsmith et al. 2013). Within different clinical conditions, such as CRPS, one or more of these

48 components of self can be disrupted (Moseley and Flor 2012). Here we are interested in self-

49 localisation.

50

51

52

There is evidence that people with CRPS have impaired self-localisation during explicit tasks.

53

For example, people with CRPS generally tend to be less accurate and slower than healthy

54 controls when instructed to match the position of one limb to a bodily movement or position performed by the opposite limb (Brun, Giorgi et al. 2019). While such findings support a generalised impairment, recent work suggests that CRPS may share features with spatial neglect, a neurological disorder arising after a lesion to the parietal cortex in which the person "neglects" the contralateral side of the world and/or of themselves, even in the absence of paralysis. For example, people suffering from neglect often show a sense of disownership (and sometimes even disgust) towards their affected limb, and this is associated with impaired self-localisation [see (Caggiano and Jehkonen 2018) for a recent systematic review]; similar features also occur in people with CRPS (Lotze and Moseley 2007, Moseley and Flor 2012). Recent work added to this picture by showing that in an implicit task involving a fake finger illusion (Walsh, Moseley et al. 2011) people with unilateral upper limb CRPS appear less affected than controls, perhaps reflecting reduced weighting of cortical networks involved in bimanual hand tasks (Wang, Butler

The idea of CRPS as a neglect-like condition, in which the side of the space usually occupied by the affected limb is affected - not just the affected limb itself - is supported by recent work. Reid and colleagues (Reid, Braithwaite et al. 2018) found that people with unilateral upper limb CRPS were less accurate in drawing consecutive circles both with their affected hand, and with their unaffected hand generating the movement in the affected side of space (i.e. arm crossed over the midline). Other experimental and clinical studies highlight the potential involvement of the side of space in CRPS. In people with unilateral CRPS, temperature changes in both the affected and unaffected limb occur when the limb is placed in the affected side of space (Moseley, Gallace et al. 2009) [although see (De Paepe, Legrain et al. 2020) for conflicting results]. Further, people with CRPS report a decrease in pain when the affected limb is positioned in the unaffected side

77 of space, both physically (Moseley, Gallace et al. 2009) and perceptually using prisms [see, for 
78

79

80

81

82

83

84

85

86

87

88

89

90

91

92

93

94

95

96

97

98

99

100

101

102

103

104

105

106

107

108

109

110

111

112

113

114

115

116

117

example, (Bultitude and Rafal 2010, Moseley, Gallace et al. 2013, Christophe, Chabanat et al. 2016)].

Self-localisation of the body requires integration and weighting of inputs from various sensory sources (e.g., the visual encoded position, proprioceptive encoded position), and is also influenced by space-specific encoding (e.g., neglect-like involvement). Impairment of tactile (Moseley, Gallagher et al. 2012, Stanton, Lin et al. 2013, Catley, O'Connell et al. 2014) and proprioceptive (Bowering, Butler et al. 2014, Stanton, Leake et al. 2016) processing has been observed in people with a range of persistent pain states, not just CRPS, and at present, it is not entirely clear what might underlie impaired self-localisation in people with CRPS or non-CRPS persistent pain. One way to delineate the influences of these features is to use sensory deception, whereby sensory input is manipulated to then determine the degree to which various inputs are relied upon to localise the limb. Such approaches can be considered to interrogate implicit mechanisms because the participant is naïve to the context of the task. A relevant sensory illusion that involves self-localisation of the hand is the Disappearing Hand trick [DHT, (Newport and Gilpin 2011)]. This illusion uses proprioceptive recalibration to induce visuoproprioceptive incongruence, such that where you see the hand to be is not where it is actually located (Bellan, Gilpin et al. 2015, Bellan, Gilpin et al. 2017). The manipulation culminates when one hand is removed from view and the participant reaches over to touch the missing hand, realising then that it is not where they thought it to be. Our past work in healthy volunteers shows that even if the final step is omitted, re-weighting of proprioceptive and visual information still gradually occurs, such that over time, they begin to localise their missing hand as being closer to the proprioceptively encoded position than the visually encoded position. That is, closer to its true position (Bellan, Gilpin et al. 2015, Bellan, Gilpin et al. 2017).

The effects of illusions in people with persistent pain problems are difficult to predict. One report suggests that people with CRPS experience pain when faced with illusions that involve sensory incongruence (Harris 1999, Brun, Giorgi et al. 2019, Brun, Mercier et al. 2019), including visual illusions such as the rabbit-duck effect (Hall, Harrison et al. 2011). Other reports offer contrary findings - when sensory incongruence is induced via a rubber hand illusion (RHI; (Botvinick and Cohen 1998), they experienced the illusion similarly to healthy volunteers and do not report increased pain (Reinersmann, Landwehrt et al. 2012, Reinersmann, Landwehrt et al. 2013). Importantly, these studies that test the response of people with CRPS to illusions or sensory conflicts have involved explicit tasks. In other words, participants know that a conflict exists, or an illusion is occurring. For example, during the RHI (Botvinick and Cohen 1998), participants are explicitly aware that the prosthetic arm is not their own arm. Unlike the RHI or doing paradoxical movements behind a mirror (McCabe, Haigh et al. 2005), the DHT leaves participants genuinely naïve to what is actually happening (Bellan, Gilpin et al. 2015, Bellan, Gilpin et al. 2017). The potential importance of testing performance during implicit tasks is quite well established in studies with people with spatial neglect [for a review see (Berti 2002)] but 
118 also, for example, with the case of cortical blindness, in which people 'see' nothing, but can 119 correctly identify the colour of a shape presented in front of them [for a review see (Hadid and 120 Lepore 2017]].

121

122

123

124

125

126

127

128

129

130

131

132

133

134

135

136

137

138

139

140

141

142

143

144

145

146

147

148

149

150

151

152

Here we used the DHT to test the primary hypothesis that participants with CRPS would be less accurate than both pain-free controls and participants with non-CRPS hand pain in localising their hand after the DHT. Our secondary hypotheses were that (1) participants with non-CRPS persistent hand pain would be less accurate than pain-free controls in hand-localisation, and (2) that participants in both persistent hand pain groups would be less accurate in localising their affected hand than in localising their unaffected hand. From herein we will consider the proprioceptively-encoded location as the 'true' location and deviation from that will be considered 'less accurate'. However, we accept that this definition serves the purpose of simplifying language while it relies on assumptions that cannot be validated.

\section{Materials \& Methods}

\section{Ethical approval}

All participants gave written consent prior to participating in the study. The study was performed in accordance with the ethical standards laid down in the 1991 Declaration of Helsinki and was approved by the Human Research Ethics Committee of the University of South Australia (ID number 0000034649).

\section{Participants}

Participants ( $\mathrm{n}=60$ ) were divided into three groups: pain-free individuals ('Control'); individuals with a diagnosis of upper limb CRPS ('CRPS'); and individuals with pain in their upper limbs for more than three months, but who did not meet the criteria for a diagnosis of CRPS ('NonCRPS Pain'). The control group comprised 29 individuals (mean age $36 \pm 19$ years, 10 males), all right-handed except for one (ambidextrous). The CRPS group comprised 16 individuals (mean age $39 \pm 12$ years, 4 males); 12 were right-handed, three left-handed and one was ambidextrous. All CRPS participants had received a formal diagnosis by their health practitioner, however a further clinical assessment of signs and symptoms was performed before commencing the experimental session. The assessment was performed following the diagnostic criteria of the International Association for the Study of Pain (IASP) (Harden, Bruehl et al. 2007) (see Table S1 in Supplemental Material). The Non-CRPS Pain group were 15 right-handed participants (mean age $58 \pm 13$ years, 2 males). For all of the participants in the CRPS and Non-CRPS Pain groups pain was located in the hand and, for most of them, the pain radiated to the entire upper limb and 
153 shoulder. A sample size calculation indicated a minimum total sample size of 36 ( $\mathrm{n}=12$ /group), 154 in order to obtain a statistical power of at least $95 \%$ for medium effect size $(f=0.25)$ and an $\alpha$ set 155 at 0.05 . The eligibility criteria for each group is listed in Table 1.

156

157

158

159

160

161

162

163

164

165

166

167

168

169

170

171

172

173

174

175

176

177

178

179

180

181

182

183

184

185

186

187

188

Insert Table 1 about here

\section{Procedure}

Participants attended the Body in Mind lab in Adelaide, Australia, for a single one-and-half-hour session. All participants were asked to provide basic demographic data and to complete four questionnaires assessing their mood [Depression Anxiety Stress Scale-21, DASS-21 (Antony, Bieling et al. 1998, Osman, Wong et al. 2012)], presence of pain catastrophizing thoughts [The Pain Catastrophizing Scale, PCS (Sullivan, Bishop et al. 1995)], and body perception disturbances [questions 1 to $6 \mathrm{~b}$ of the Bath CRPS Body Perception Disturbance Scale, BPDS (Lewis and McCabe 2010); question 7 was excluded because it could not be administered online, with minimal involvement of the experimenters]. Participants belonging to the pain groups (CRPS and non-CRPS Pain) were asked to provide details concerning their condition (see Table $\mathrm{S} 2$ ).

\section{Self-localisation}

All participants performed a hand-localisation task in three different conditions, repeated twice (once for each hand, for a total of six trials), using the MIRAGE Multisensory Illusion Box (Newport, Pearce et al. 2010). Participants put their hands inside the machine and, when looking down, they saw a real time video image of their hands that was either manipulated (in the DHT condition) or not (in the congruent conditions). Specifically, during the DHT condition, participants underwent the adaptation phase of an illusion called the Disappearing Hand Trick (DHT) (Newport and Gilpin 2011). During this phase, unbeknownst to the participants, a mismatch is induced between the visually-encoded (i.e. where the participants see their hands to be) and the proprioceptively-encoded (i.e. where their hands really are) position of the hands. This leads to a localisation weighting that is biased towards the visual representation of the hand. Therefore, the participants see their hands moving inwards, while in fact, they have to move their hands outwards to keep the visual image of their hands central (see Manipulation Check below for more information).

During the two control conditions, the visually and proprioceptively encoded positions of the hand were congruent, such that the hands were exactly where the participants saw them to be. That is, during the Hidden Hand (HH) condition, the participants saw their hands moving outwards, and no manipulation was performed (i.e. the hands were actually moving outwards), 
189

190

191

192

193

194

195

196

197

198

199

200

201

202

203

204

205

206

207

208

209

210

211

212

213

214

215

216

217

218

219

220

221

222

while, during the Static Hand (SH) condition, the hands just hovered over the surface of the table inside the machine, staying centralised.

In all conditions, after 25 seconds (during which the participants either moved their hands or kept them still), they were asked to rest the hands on the bottom of the machine and to keep looking at one of their hands, while it disappeared (a black square was superimposed to the image of their hand). Once the hand was out of view, a localisation task commenced: a red arrow appeared in the middle of the screen and started travelling at a constant speed towards the disappeared hand. The participants were asked to look at the side of the space where the hand was to be located and to keep track of where they felt their hand to be and say "stop" when they thought the arrow was pointing at their middle finger. For each trial, seven localisations were performed (one every 15 seconds). Then, both hands disappeared, and the actual location of the target hand was recorded as the baseline measure (see Fig. S1 in Supplemental Material). Further details about the procedure and a video depicting the movement of the hands can be found elsewhere (Bellan, Gilpin et al. 2015, Bellan, Gilpin et al. 2017).

\section{Swelling and pain}

At the beginning of the experimental session and after each trial, the experimenter measured the circumference of the participants' second and third fingers, and wrists of both hands, in order to assess any swelling change. In addition, participants belonging to the pain groups were asked to rate their pain on a scale between 0 (no pain at all) and 10 (worst pain imaginable).

\section{Manipulation check - Disappearing Hand Trick}

During the DHT (Newport and Gilpin 2011), the adaptation phase explained above for the DHT condition was performed and, subsequently, after one hand disappeared, the participants are asked to reach across with their other hand to touch their disappeared hand. This "full DHT" (participants aware that that their hand was not where they thought it was) was performed to verify whether the participants noticed any differences between the experimental and the control conditions. Specifically, if the illusion worked and the participants failed to touch their disappeared hand, they would be expected to show surprise and they were then asked whether they realised that their hands were not where they thought they were. All the participants were surprised and, when asked whether they noticed any difference between the conditions administered during the experiment, none of them were able to tell the difference between the control and the experimental conditions. 


\section{Statistical analysis}

224 Data were inspected and analysed by using custom-made scripts in RStudio version 1.2.1335

225 (RStudio Team, 2018) for Windows. The lme4 package was used for linear mixed-effects models

226 (LMM) (Bates, Maechler et al. 2015). Different packages from the Tidyverse collection

227 (Wickham, Averick et al. 2019) were used to explore and plot the data.

228 Akaike Information Criterion [AIC; (Akaike 1974)], Bayesian Information Criterion [BIC;

229 (Schwarz 1978)] and log-likelihood were used to assess the fit of the LMM.

230

231 Preliminary analysis

232 Data were visually inspected according to the two main hypotheses.

233

234 Data handling

235 For each participant, localisation error was calculated as the difference (in pixels) between the

236

237

238

239

240

241

242

243

244

245

246

247

248

249

250

251

252

253 actual position of the hand and the position of the hand as indicated by the participants at each point in time (i.e. where the participants stopped the arrow). We chose pixel as unity of measure because the error was measured directly on the screen where both the arrow and the hand appeared. The localisation error indicates the accuracy in localising one's own limb, with higher scores suggesting a larger discrepancy between the actual and perceived position of the limb, and, therefore, lower accuracy.

\section{Primary hypothesis and secondary hypothesis 1: LMM1}

According to the primary hypothesis, participants in the CRPS group would show larger localisation error ('Error') than Controls and the Non-CRPS Pain group; at the same time, according to the first secondary hypothesis, participants in the Non-CRPS Pain group would in turn show larger localisation error ('Error') than Controls. The effects of 'Time' $\left(\mathrm{T}_{1}\right.$ to $\left.\mathrm{T}_{7}\right)$ and 'Condition' (DHT, Hidden, Static) on 'Error' were plotted for each participant. High interindividual variability meant that the factor 'Participants' was included in the final model as random factor, with 'Condition', 'Time' as fixed effects, and 'Error' as the dependent variable (see Fig. S2). In line with previous results (Bellan, Gilpin et al. 2015, Bellan, Gilpin et al. 2017) we expect a main effect of Time for all groups.

Peer] reviewing PDF | (2020:11:55593:1:1:NEW 22 Jun 2021) 
254 Secondary hypothesis 2 and exploratory analysis: LMM2

255 According to the Secondary hypothesis 2, participants with hand pain would show larger

256 localisation error when judging location of their affected than their unaffected limb. The

257 difference in the average localisation error over $\mathrm{T} 1$ to $\mathrm{T} 7 \mathrm{between}$ limbs performed by both

258 groups for each condition was plotted. Because a difference was found to exist between the

259 localisation of the affected and unaffected hand within the DHT condition, the decision was

260 made to include the factor Hand (affected, unaffected) in the second model.

261 Hand laterality was considered as a covariate. In this regard, previous research (Grabherr, Russek

262 et al. 2019) showed no difference in localisation error between the right and the left hand, in

263 right-handed participants. The current study involved two clinical groups with hand pain, so an

264 effect of handedness (right, left) would have been difficult to interpret, because either the right or

265 the left hand could have also been the affected. Therefore, in order to still account for the effect

266 of handedness, the factor 'Hand' (affected, unaffected) was only included in the second LMM, in

267 which only participants in the CRPS and non-CRPS Pain groups were included.

268 Finally, in the pain groups separately, Error was plotted against: Age, Sex, change in Swelling

269 and Pain ratings (both calculated as the difference between baseline and after each condition),

270 DASS scores, PCS score, BPDS scores, and Duration of the painful condition (in months).

271 Because no specific hypotheses were made for these comparisons, after visual inspection of the

272 data, the factors 'Age' (years) and 'Anxiety' (the score of anxiety from the DASS questionnaire)

273 were selected to be included in the second LMM.

\section{Results}

\section{Localisation error}

\section{Effect of group on localisation error (Primary Hypothesis and Secondary}

277 Hypothesis 1)

278 All significant effects and interactions of the LMM as well as the model fit of localisation error

279 are included in Table S3.

280 LMM results indicated a main effect of Condition $[\mathrm{F}(2,2460)=1040.326, p<0.001]$, revealing that 281 participants were significantly more inaccurate (larger localisation error) in the DHT condition $282(\mathrm{M}=100.71, \mathrm{SD}=39.67$ pixels $)$ than in the two control conditions [i.e. Hidden $(\mathrm{M}=20.95$, SD $283=22.05$ pixels $)$ and Static $(\mathrm{M}=20.78, \mathrm{SD}=17.26$ pixels $)]$, regardless of group. In addition, 284 participants' accuracy varied significantly over consecutive localisations, as indicated by a main 285 effect of Time $[\mathrm{F}(1,2460)=22.56, p<0.001]$. Specifically, significant interactions between Time 286 and Condition $(p<0.001)$ revealed that participants became more accurate over time during the 287 DHT condition but not during the control conditions, which reflected the large initial error in the 288 DHT condition but not in the control conditions (see Fig. 1). 
289

290

291

292

293

294

295

296

297

298

299

300

301

302

303

304

305

306

307

308

309

310

311

312

313

314

315

316

317

318

319

320

321

322

323

324

325

Insert Fig. 1 about here

Finally, there was no effect of Group ( $p=0.54)$, suggesting that, overall, CRPS participants were not less accurate than the other two groups, which was contrary to our prediction (see Fig. 2). However, a significant interaction between Group (non-CRPS Pain) and Time $(p<0.001)$, and between Group, Time and Condition $(p=0.002)$ suggested that the localisation error in the DHT condition decreased more rapidly in the non-CRPS Pain group than it did in the other two groups (see Fig. 1).

\section{Insert Fig. 2 about here}

\section{Influence of affected hand on localisation error in those with persistent hand pain}

\section{(Secondary Hypothesis 2)}

All significant effects and interactions of the LMM as well as the model fit of localisation error are included in Table S4.

The LMM showed a main effect of Condition $[\mathrm{F}(2,1271)=8.62, p<0.001]$, replicating the results reported above, with the largest error in the DHT condition $(\mathrm{M}=94.8, \mathrm{SD}=40.38 \mathrm{px}$; Hidden: $\mathrm{M}=21.68, \mathrm{SD}=25.48 \mathrm{px}$; Static: $\mathrm{M}=23.45, \mathrm{SD}=18.9 \mathrm{px}$ ). A significant interaction between Condition and Group ( $p=0.02$ ) also suggested that the non-CRPS Pain group showed a significantly smaller difference between DHT and the control conditions than the CRPS group did (see Table 2).

\section{Insert Table 2 about here}

Furthermore, a second significant interaction between Affected Hand and DHT Condition $(p=0.03)$ suggested larger error for the affected hand than the unaffected hand during the DHT condition than during the control conditions (Fig. 3, Table 3).

Insert Fig. 3 about here

Insert Table 3 about here

\section{Exploratory analyses}

A significant interaction between DHT Condition and Age $(p<0.001)$ showed that older age was correlated with smaller error in the DHT condition (the older the age, the smaller the error), while the opposite was true for the Static and Hidden conditions (Fig. 4a). The same result was 
326

327

328

329

330

331

332

333

334

335

336

337

338

339

340

341

342

343

344

345

346

347

\section{8}

349

350

351

352

353

354

355

356

357

358

359

360

361

362

363

364

365

also shown in the significant interaction between Group, Condition and Age ( $p=0.007)$, whereby the non-CRPS Pain group showed a stronger difference between the two control conditions and the DHT condition in the same direction (with positive correlation between Age and Error in the control conditions, and a negative correlation for the DHT condition) than the CRPS group did (Fig. 4b) (although note that participants in the non-CRPS Pain group were older than participants in the CRPS group). Finally, the same significant trend $(p=0.02)$ was found in the comparison between the affected and unaffected hands, showing that the affected hand showed a stronger difference than the unaffected hand between conditions in the same direction (where a positive correlation was found between Age and Error in the control conditions, and a negative correlation for the DHT condition) (Fig. 4c).

\section{Insert Fig. 4 about here}

Our exploratory analyses showed a significant interaction between Group, Condition and Anxiety $(p<0.001)$ such that in the non-CRPS pain group higher anxiety was significantly and positively correlated with larger error only in the DHT condition $(p=0.04)$, with the two control conditions showing the opposite trend (i.e. the higher the anxiety level, the smaller the error). In the CRPS group, higher anxiety was positively correlated with larger error for all three conditions, however this correlation was not significant (Fig. 5).

\section{Insert Fig. 5 about here}

\section{Discussion}

This study evaluated hand-localisation accuracy in people living with upper limb CRPS, by comparing their performance to that of a clinical control group (people living with non-CRPS persistent hand pain), and with a non-clinical control group (pain-free individuals). Our hypotheses that participants with CRPS would be less accurate in hand-localisation than painfree controls and participants with pain of other origins, and that all participants with persistent hand pain would be less accurate than pain-free controls were not supported. With regards to our Secondary hypothesis 1 , we found the opposite of our prediction - people living with non-CRPS hand pain showed a quicker re-weighting toward proprioception after the DHT than the other two groups did - possibly indicating a smaller susceptibility to the illusion. Our Secondary hypothesis 2 was partially supported - participants living with hand pain were less accurate in localising their affected than their unaffected hand, but only in the DHT condition, involving incongruent proprioceptive and visual encoded representations. Crucially, no significant difference between the groups or between the affected and unaffected hands was found for the control conditions, suggesting that, under normal circumstances (when accurate visual and proprioceptive cues are provided) persistent pain, whether in association with CRPS or not, does not appear to affect the self-localisation accuracy of the affected body part. 
366 People with CRPS tend to report difficulties in localising their own affected limb when they

367

368

369

370

371

372

373

374

375

376

377

378

379

380

381

382

383

384

385

386

387

388

389

390

391

392

393

394

395

396

397

398

399

400

401

402

403

404

405

cannot see it. This has been anecdotally reported and experimentally studied under different conditions (Lewis and McCabe 2010, Lewis, Kersten et al. 2010, Lewis and McCabe 2010, Reinersmann, Landwehrt et al. 2012). Several studies found that these people are less accurate than those without CRPS in tasks in which an accurate spatial representation of the limb is required [e.g. (Reid, Braithwaite et al. 2018, Brun, Giorgi et al. 2019, Brun, Mercier et al. 2019)]. A range of other findings point to a kind of spatial (Galer and Jensen 1999), or so-called 'somatospatial' neglect (Reid, Wallwork et al. 2016). However, this idea remains controversial because the total picture is confusing. For example, a very recent study (De Paepe, Legrain et al. 2020) failed to replicate previous studies that indicated the presence of a perceptual bias away from the affected limb during a temporal order judgement task in CRPS participants [see also (Bultitude, Walker et al. 2017)]. The present results add weight to the argument against neglectlike aspects of CRPS because, if proprioceptive encoded location had less weighting in CRPS, then the error during the DHT condition would have been greater in the CRPS group than it was in the other groups, which was not the case.

Finally, and crucial for the present study, vast literature has now shown the dissociation between implicit and explicit processing of spatial representation in studies involving people with neglect. For example, people with neglect show a dissociation in accessing the mental number line: they fail to access it explicitly, but they are accurate if asked to do so via an implicit task by using an alternative strategy that does not involve the damaged brain areas (Priftis, Zorzi et al. 2006). Even if the neglect-like metaphor stands true for people with CRPS, this possible dissociation needs to be considered. The peculiarity of the illusion employed in this study is that participants rarely realise they have been tricked and, if they do realise, it only happens after they fail in an attempt to physically locate their hand using their other hand. This means that, at least in part, the localisation task (which precedes the physical attempt to touch the disappeared hand) is conducted under the assumption that the hand is where the participant last saw it. Therefore, the participants are led to believe they are doing an explicit localisation task, while, in fact, they are not. Future studies will need to further investigate the possibility of a dissociation between implicit and explicit tasks by possibly using paradigms employed in the study of people with neglect.

Interpretation of the current findings should consider the likely underpinnings of the illusion we used. The DHT relies on visually encoded data having greater weighting than proprioceptively encoded data, and thus the finally perceived location of the hand reflects the former more closely than the latter. That the error was greater for the affected hand than it was for the unaffected hand for both persistent hand pain groups (i.e. the illusion was 'stronger') implies that the extent to which visually encoded data outweighs proprioceptively encoded data is greater for the affected than the unaffected hand in these groups. This would be predicted on the basis of use-

Peer) reviewing PDF | (2020:11:55593:1:1:NEW 22 Jun 2021) 
406 dependency of neural networks: using the affected hand less than the unaffected one could 407 explain our results, and might be mediated by impaired proprioception. Relevant here is a study 408 in people with CRPS that aimed to interrogate the relative weighting of bimanual cortical 409 representations (i.e. neural networks that underpin two-handed tasks) and unimanual cortical 410 representations (i.e. those that underpin one-handed tasks), through a procedure that induces the 411 illusion that one's index fingers are closer to each other than they really are (Walsh, Moseley et 412 al. 2011, Wang, Butler et al. 2019). That study showed a smaller illusion in people with CRPS 413 than in healthy controls, which implies that the extent to which bimanual cortical representations outweigh unimanual cortical representations is less in people with CRPS than it is in healthy controls. Again, this result would be predicted on the basis of use-dependency of neural networks: avoiding bimanual tasks could explain that result. That people with CRPS perform normally on the RHI (Botvinick and Cohen 1998, Reinersmann, Landwehrt et al. 2013) does not contradict our evidence of altered response on the localisation task, because the RHI involves a different mechanism - synchronous visual and tactile input allocated by the brain to the same event.

421

422

The current results raise interesting reflections on why people with CRPS-related persistent pain in particular can report 'losing track of their hand' during daily living. Perhaps our results offer a potential explanation. That is, without using the hand, or indeed even looking at it, prevents the usual opportunities for brain-held models of the body and its location to be updated. That patients report that seeing their hand surprises them because they thought it was somewhere else, not because they thought it was missing, points to this failure to update. Although speculative, this scenario shares parallels with asomatognosia, where brain damage affecting proprioceptive or motor function, combined with not looking at a hand, can lead to a failure to recognise a limb or part thereof (Mendoza 2011).

Older people were both less accurate during control tasks (when visually encoded data were not manipulated) and more accurate during the DHT. This interesting finding also raises an important caution because it opens the possibility that age of participants may have contributed to the non-CRPS hand pain group showing a quicker relocalisation than the other groups. Why might age affect performance in this way? Perhaps proprioceptively encoded models of the body continue to improve with age. This would mean that, when vision is removed, older people update their internal model of body position more quickly, as we saw here. However, this would also result in more accurate performance in control tasks, which we did not observe.

Alternatively, age is known to be associated with decreasing reliability of visually encoded data (Kolesnikov, Fan et al. 2010), which would explain less accuracy during control tasks, smaller illusion effect during the DHT, and more rapid recovery after the DHT. In addition, Laurienti and colleagues (2006) reported that older people showed enhanced multisensory integration: this population show a better performance when there are two stimuli providing the same information than when a single stimulus is providing it. The authors of that paper linked this finding to an 
446 offset decline in signal-to-noise-ratio with increasing age. It is thus interesting to consider that

447 this enhanced integration might also involve enhanced detection of multisensory incongruence.

448 Clearly, more research is required to understand this interesting finding.

449

450

451

452

453

454

455

456

457

458

459

460

461

462

463

464

465

466

467

468

469

470

471

472

473

474

\section{Conclusions}

476

477 In conclusion, contrary to our hypotheses, being in pain does not seem to necessarily lead to 478

479

480

481

482

Finally, exploratory analysis seems to suggest that our results cannot be explained by sex or mood. Although accuracy tended to be worse in those who were more anxious according to the DASS-21, there seemed to be no specific effect on the outcome of the DHT. Another visuotactile illusion in which visual input of touch to the affected hand causes pain in people with CRPS (Acerra and Moseley 2005) but not people with non-CRPS neuropathic pain (Krämer, Seddigh et al. 2008), and other disruptions of bodily awareness are thought to be related to levels of distress (Breimhorst, Dellen et al. 2018). That finding might imply a role for mood in problems integrating bodily data in CRPS, at least in what might be called 'explicit' problems of bodily awareness. This study, alongside that of Wang et al. (2019), early studies involving implicit motor imagery (Schwoebel, Boronat et al. 2002, Moseley 2004), and a finding of a lower heartbeat-evoked potential amplitude in people with CRPS than in healthy controls (Solcà, Park et al. 2020) all involve implicit mechanisms, not explicit.

Interpretation of the current work should consider its limitations. First, we did not lodge and lock our protocol and statistical analysis plan prior to data collection. When we commenced this study, such practice was uncommon in our field, but now it is recommended, and our group is among those at the forefront of this push (Lee, Lamb et al. 2018). Failure to do this clearly represents a shortcoming in transparency and reporting. Other limitations include the age disparity between the groups, with the non-CRPS Pain group being on average older than the other two groups. In addition, the participants in that group reported a much longer duration of the painful condition. The amount of time spent being in pain might have an effect on physiological (e.g. cortical reorganization) as well psychological processes (e.g. level of distress). Finally, we interpret the results as reflecting changes in the relative weighting of visual and proprioceptive-encoded data, but we did not explicitly assess either. worst self-localization abilities. However, possibly due to use-dependent effects, people tend to perform better with the unaffected hand compared to the affected counterpart. However, future studies will need to clarify whether this disadvantage of the affected hand is body-centered or body part-centered. Interestingly, the similar performance in localization abilities of people with CRPS and pain-free controls led to the interpretation of a possible dissociation between implicit

Peer) reviewing PDF | (2020:11:55593:1:1:NEW 22 Jun 2021) 
483 and explicit neural processes in CRPS. This would once again suggest the existence of neglect484 like characteristics in CRPS.

485

486

487

488

489

490

491

492

493

494

495

496

497

498

499

500

501

502

503

504

505

506

507

508

509

510

511

512

513

514

515

516

517

518

519

520

521

522

\section{Acknowledgements}

The authors would like to thank Ms. Niki Najafi and Ms. Brooke Wilkin for their help during testing procedures. In addition, we would like to thank all the participants that volunteered their time to take part in this study. Finally, a special thanks to the Australian CRPS community.

\section{References}

Acerra, N. E. and G. L. Moseley (2005). "Dysynchiria: Watching the mirror image of the unaffected limb elicits pain on the affected side." Neurology 65(5): 751-753.

Akaike, H. (1974). "A new look at the statistical model identification." IEEE transactions on automatic control 19(6): 716-723.

Antony, M. M., P. J. Bieling, B. J. Cox, M. W. Enns and R. P. Swinson (1998). "Psychometric properties of the 42-item and 21-item versions of the Depression Anxiety Stress Scales in clinical groups and a community sample." Psychological assessment 10(2): 176.

Bates, D., M. Maechler, B. Bolker, S. Walker, R. H. B. Christensen, H. Singmann, B. Dai, G. Grothendieck, P. Green and M. B. Bolker (2015). "Package 'Ime4'." Convergence 12(1): 2.

Bellan, V., H. R. Gilpin, T. R. Stanton, L. K. Dagsdottir, A. Gallace and G. Lorimer Moseley (2017). "Relative contributions of spatial weighting, explicit knowledge and proprioception to hand localisation during positional ambiguity." Exp Brain Res 235(2): 447-455.

Bellan, V., H. R. Gilpin, T. R. Stanton, R. Newport, A. Gallace and G. L. Moseley (2015). "Untangling visual and proprioceptive contributions to hand localisation over time." Exp Brain Res 233(6): 1689-1701. Berti, A. (2002). "6.1 Unconscious processing in neglect." The cognitive and neural bases of spatial neglect: 313.

Botvinick, M. and J. Cohen (1998). "Rubber hands' feel'touch that eyes see." Nature 391(6669): 756-756 \%@ 0028-0836.

Bowering, K. J., D. S. Butler, I. J. Fulton and G. L. Moseley (2014). "Motor Imagery in People With a History of Back Pain, Current Back Pain, Both, or Neither." The Clinical Journal of Pain 30(12): 1070-1075. Breimhorst, M., C. Dellen, M. Wittayer, C. Rebhorn, P. D. Drummond and F. Birklein (2018). "Mental load during cognitive performance in complex regional pain syndrome I." Eur J Pain 22(7): 1343-1350. Brun, C., N. Giorgi, A.-M. Pinard, M. Gagné, C. S. McCabe and C. Mercier (2019). "Exploring the relationships between altered body perception, limb position sense, and limb movement sense in Complex Regional Pain Syndrome." The Journal of Pain 20(1): 17-27.

Brun, C., C. Mercier, S. Grieve, S. Palmer, J. Bailey and C. S. McCabe (2019). "Sensory disturbances induced by sensorimotor conflicts are higher in complex regional pain syndrome and fibromyalgia 
523 compared to arthritis and healthy people, and positively relate to pain intensity." European Journal of 524 Pain 23(3): 483-494.

525 Bultitude, J. H. and R. D. Rafal (2010). "Derangement of body representation in complex regional pain 526 syndrome: report of a case treated with mirror and prisms." Experimental brain research 204(3): 409-

527418.

528 Bultitude, J. H., I. Walker and C. Spence (2017). "Space-based bias of covert visual attention in complex

529 regional pain syndrome." Brain 140(9): 2306-2321.

530 Caggiano, P. and M. Jehkonen (2018). "The 'Neglected' Personal Neglect." Neuropsychology review

531 28(4): 417-435.

532 Catley, M. J., N. E. O'Connell, C. Berryman, F. F. Ayhan and G. L. Moseley (2014). "Is tactile acuity altered

533 in people with chronic pain? a systematic review and meta-analysis." J Pain 15(10): 985-1000.

534 Christophe, L., E. Chabanat, L. Delporte, P. Revol, P. Volckmann, S. Jacquin-Courtois and Y. Rossetti

535 (2016). "Prisms to shift pain away: pathophysiological and therapeutic exploration of CRPS with prism

536 adaptation." Neural plasticity 2016.

537 De Paepe, A., V. Legrain, L. Van der Biest, N. Hollevoet, A. Van Tongel, L. De Wilde, H. Jacobs and G.

538 Crombez (2020). "An investigation of perceptual biases in complex regional pain syndrome." PeerJ

539 8(e8819).

540 Galer, B. S. and M. Jensen (1999). "Neglect-like symptoms in complex regional pain syndrome: results of

541 a self-administered survey." Journal of pain and symptom management 18(3): 213-217.

542 Grabherr, L., L. N. Russek, V. Bellan, M. Shohag, D. Camfferman and G. L. Moseley (2019). "The

543 disappearing hand: vestibular stimulation does not improve hand localisation." PeerJ 7: e7201.

544 Hall, J., S. Harrison, H. Cohen, C. S. McCabe, N. Harris and D. R. Blake (2011). "Pain and other symptoms

545 of CRPS can be increased by ambiguous visual stimuli-an exploratory study." European Journal of Pain

546 15(1): 17-22.

547 Harden, R. N., S. Bruehl, M. Stanton-Hicks and P. R. Wilson (2007). "Proposed new diagnostic criteria for

548 complex regional pain syndrome." Pain Med 8(4): 326-331.

549 Harris, A. J. (1999). "Cortical origin of pathological pain." Lancet 354(9188): 1464-1466.

550 Kolesnikov, A. V., J. Fan, R. K. Crouch and V. J. Kefalov (2010). "Age-Related Deterioration of Rod Vision

551 in Mice." Journal of Neuroscience 30(33): 11222-11231.

552 Krämer, H. H., S. Seddigh, G. Lorimer Moseley and F. Birklein (2008). "Dysynchiria is not a common

553 feature of neuropathic pain." Eur J Pain 12(1): 128-131.

554 Lee, H., S. E. Lamb, M. K. Bagg, E. Toomey, A. G. Cashin and G. L. Moseley (2018). "Reproducible and

555 replicable pain research: a critical review." Pain 159(9): 1683-1689.

556 Lewis, J. and C. McCabe (2010). "Body perception disturbance (BPD) in CRPS." Practical Pain

557 Management: 60-66.

558 Lewis, J. S., P. Kersten, C. S. McCabe, K. M. McPherson and D. R. Blake (2007). "Body perception

559 disturbance: a contribution to pain in complex regional pain syndrome (CRPS)." Pain 133(1-3): 111-119.

560 Lewis, J. S., P. Kersten, K. M. McPherson, G. J. Taylor, N. Harris, C. S. McCabe and D. R. Blake (2010).

561 "Wherever is my arm? Impaired upper limb position accuracy in complex regional pain syndrome." Pain

562 149(3): 463-469.

563 Lewis, J. S. and C. S. McCabe (2010). "Body Perception Disturbance (BPD) in CRPS." Practical PAIN

564 MANAGEMENT 3(3): 60-66.

565 Lotze, M. and G. L. Moseley (2007). "Role of distorted body image in pain." Curr Rheumatol Rep 9(6):

566 488-496.

567 McCabe, C. S., R. C. Haigh, P. W. Halligan and D. R. Blake (2005). "Simulating sensory-motor

568 incongruence in healthy volunteers: implications for a cortical model of pain." Rheumatology (Oxford)

569 44(4): 509-516. 
570 Mendoza, J. E. (2011). Asomatognosia. Encyclopedia of Clinical Neuropsychology. J. S. Kreutzer, J.

571 DeLuca and B. Caplan. New York, NY, Springer New York: 257-258.

572 Moseley, G. L. (2004). "Why do people with complex regional pain syndrome take longer to recognize

573 their affected hand?" Neurology 62(12): 2182-2186.

574 Moseley, G. L. and H. Flor (2012). "Targeting cortical representations in the treatment of chronic pain: a

575 review." Neurorehabilitation and neural repair 26(6): 646-652.

576 Moseley, G. L., A. Gallace, F. Di Pietro, C. Spence and G. D. lannetti (2013). "Limb-specific autonomic

577 dysfunction in complex regional pain syndrome modulated by wearing prism glasses." PAIN $^{\circledR}$ 154(11):

578 2463-2468.

579 Moseley, G. L., A. Gallace and C. Spence (2009). "Space-based, but not arm-based, shift in tactile

580 processing in complex regional pain syndrome and its relationship to cooling of the affected limb." Brain

581 132(Pt 11): 3142-3151.

582 Moseley, G. L., L. Gallagher and A. Gallace (2012). "Neglect-like tactile dysfunction in chronic back pain."

583 Neurology 79(4): 327-332.

584 Newport, R. and H. R. Gilpin (2011). "Multisensory disintegration and the disappearing hand trick."

585 Current Biology 21(19): R804-R805.

586 Newport, R., R. Pearce and C. Preston (2010). "Fake hands in action: embodiment and control of

587 supernumerary limbs." Exp Brain Res 204(3): 385-395.

588 Osman, A., J. L. Wong, C. L. Bagge, S. Freedenthal, P. M. Gutierrez and G. Lozano (2012). "The depression

589 anxiety stress Scales-21 (DASS-21): further examination of dimensions, scale reliability, and correlates."

590 Journal of clinical psychology 68(12): 1322-1338.

591 Priftis, K., M. Zorzi, F. Meneghello, R. Marenzi and C. Umiltà (2006). "Explicit versus implicit processing of

592 representational space in neglect: dissociations in accessing the mental number line." $\underline{\text { J Cogn Neurosci }}$

593 18(4): 680-688.

594 Reid, E., S. B. Wallwork, D. Harvie, K. J. Chalmers, A. Gallace, C. Spence and G. L. Moseley (2016). "A New

595 Kind of Spatial Inattention Associated With Chronic Limb Pain?" Ann Neurol 79(4): 701-704.

596 Reid, E. J., F. A. Braithwaite, S. B. Wallwork, D. Harvie, K. J. Chalmers, C. Spence, A. Gallace and G. L.

597 Moseley (2018). "Spatially-defined motor deficits in people with unilateral complex regional pain

598 syndrome." Cortex 104: 154-162.

599 Reinersmann, A., J. Landwehrt, E. K. Krumova, S. Ocklenburg, O. Gunturkun and C. Maier (2012).

600 "Impaired spatial body representation in complex regional pain syndrome type 1 (CRPS I)." Pain 153(11):

601 2174-2181.

602 Reinersmann, A., J. Landwehrt, E. K. Krumova, J. Peterburs, S. Ocklenburg, O. Gunturkun and C. Maier

603 (2013). "The rubber hand illusion in complex regional pain syndrome: preserved ability to integrate a

604 rubber hand indicates intact multisensory integration." Pain 154(9): 1519-1527.

605 Schwarz, G. (1978). "Estimating the dimension of a model." The annals of statistics 6(2): 461-464.

606 Schwoebel, J., C. B. Boronat and H. Branch Coslett (2002). "The man who executed "imagined"

607 movements: evidence for dissociable components of the body schema." Brain Cogn 50(1): 1-16.

608 Serino, A., A. Alsmith, M. Costantini, A. Mandrigin, A. Tajadura-Jimenez and C. Lopez (2013). "Bodily

609 ownership and self-location: components of bodily self-consciousness." Conscious Cogn 22(4): 1239-

6101252.

611 Solcà, M., H. D. Park, F. Bernasconi and O. Blanke (2020). "Behavioral and neurophysiological evidence

612 for altered interoceptive bodily processing in chronic pain." Neurolmage: 116902.

613 Stanton, T. R., H. B. Leake, K. J. Chalmers and G. L. Moseley (2016). "Evidence of Impaired Proprioception

614 in Chronic, Idiopathic Neck Pain: Systematic Review and Meta-Analysis." Phys Ther 96(6): 876-887.

615 Stanton, T. R., C.-W. C. Lin, H. Bray, R. J. E. M. Smeets, D. Taylor, R. Y. W. Law and G. L. Moseley (2013).

616 "Tactile acuity is disrupted in osteoarthritis but is unrelated to disruptions in motor imagery

617 performance." Rheumatology 52(8): 1509-1519. 
618 Sullivan, M. J., S. R. Bishop and J. Pivik (1995). "The pain catastrophizing scale: development and 619 validation." Psychological assessment 7(4): 524.

620 Walsh, L. D., G. L. Moseley, J. L. Taylor and S. C. Gandevia (2011). "Proprioceptive signals contribute to 621 the sense of body ownership." The Journal of physiology 589(12): 3009-3021.

622 Walsh, L. D., G. L. Moseley, J. L. Taylor and S. C. Gandevia (2011). "Proprioceptive signals contribute to 623 the sense of body ownership." J Physiol 589(Pt 12): 3009-3021.

624 Wang, A. P., A. A. Butler, J. D. Valentine, C. D. Rae, J. H. McAuley, S. C. Gandevia and G. L. Moseley 625 (2019). "A Novel Finger Illusion Reveals Reduced Weighting of Bimanual Hand Cortical Representations 626 in People With Complex Regional Pain Syndrome." J Pain 20(2): 171-180.

627 Wickham, H., M. Averick, J. Bryan, W. Chang, L. McGowan, R. François, G. Grolemund, A. Hayes, L. Henry 628 and J. Hester (2019). "Welcome to the Tidyverse." Journal of Open Source Software 4(43): 1686. 


\section{Figure 1}

Error by Time by Condition by Group

Fig. 1 - The dots represents each localisation made by the each participant in each group. In the DHT condition (in red) all participants tend to start with a larger error compared to the other two conditions, but they become more accurate by time, even though the error never reaches 0 (i.e. no participants was able to correctly localise their hand). Also, the steepness of the red lines support the idea that participants in the Non-CRPS Pain group were probably faster in their reliance on proprioception, as shown by a steeper line towards 0 , with an initial intercept similar to the other two groups. 
Figure 1: Error by time by condition by group

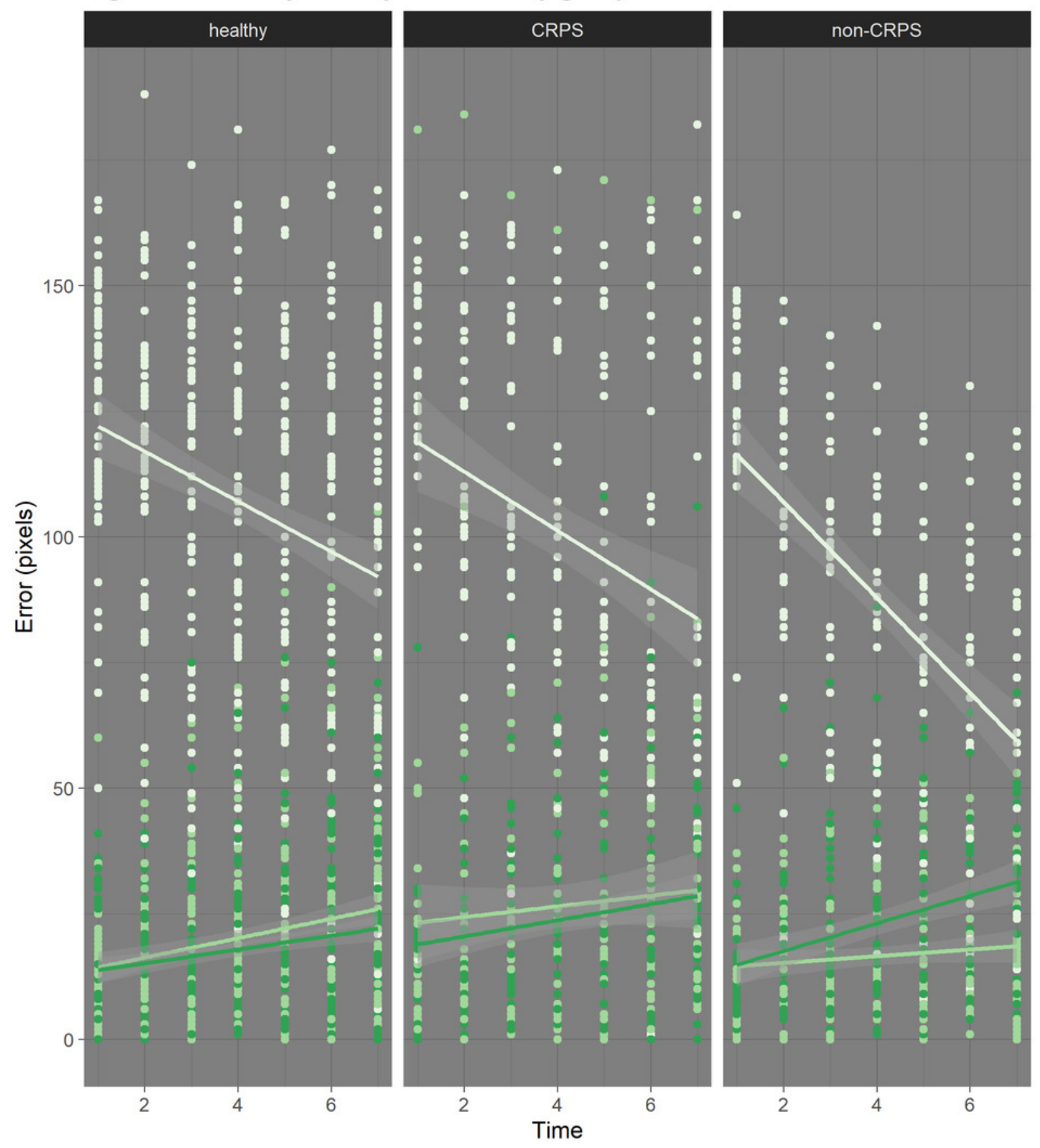

condition

DHT Hidden Static 


\section{Figure 2}

Error by Condition by Group

Fig. 2 - The raincloud figure represents the distribution of the error (y axis) for each participant and for each condition ( $x$ axis). The groups are colour coded. Even though the distribution is quite similar across different group, the CRPS group seems to have a larger variability across participants (e.g. each participant seems to behave quite differently compared to the others within the same group). However, contrary to our prediction, the participants in the CRPS groups were not less accurate overall compared to the other groups. 
Figure 2: Error by condition by group

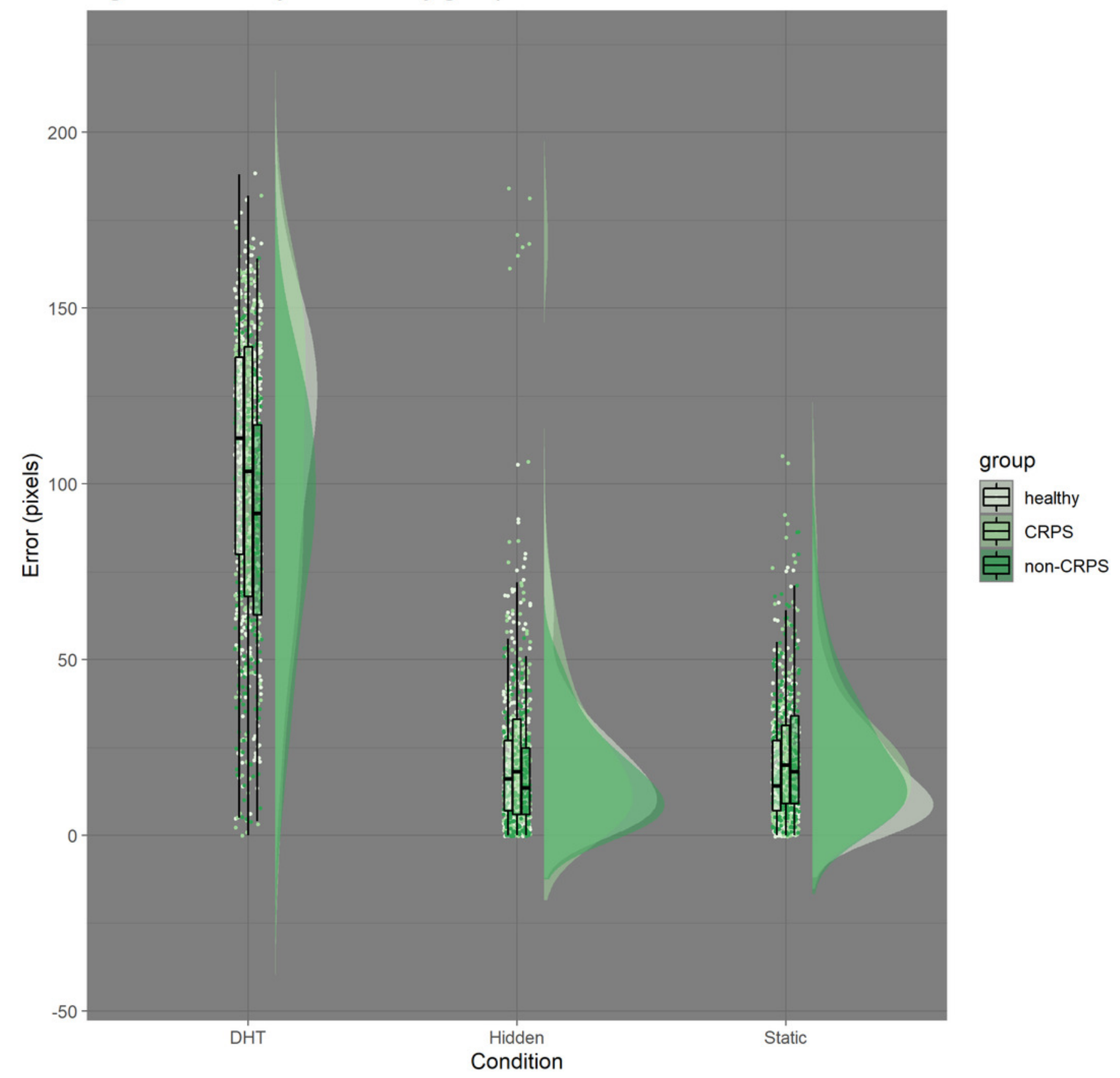


Figure 3

Error by Condition by Hand

Fig. 3 - Both groups show larger localisation errors for the DHT condition compared to the other two control conditions. However the difference between Affected and Unaffected hand is smaller in the non-CRPS than in the CRPS group. 
Figure 3: Error by condition by hand (Pain groups only)

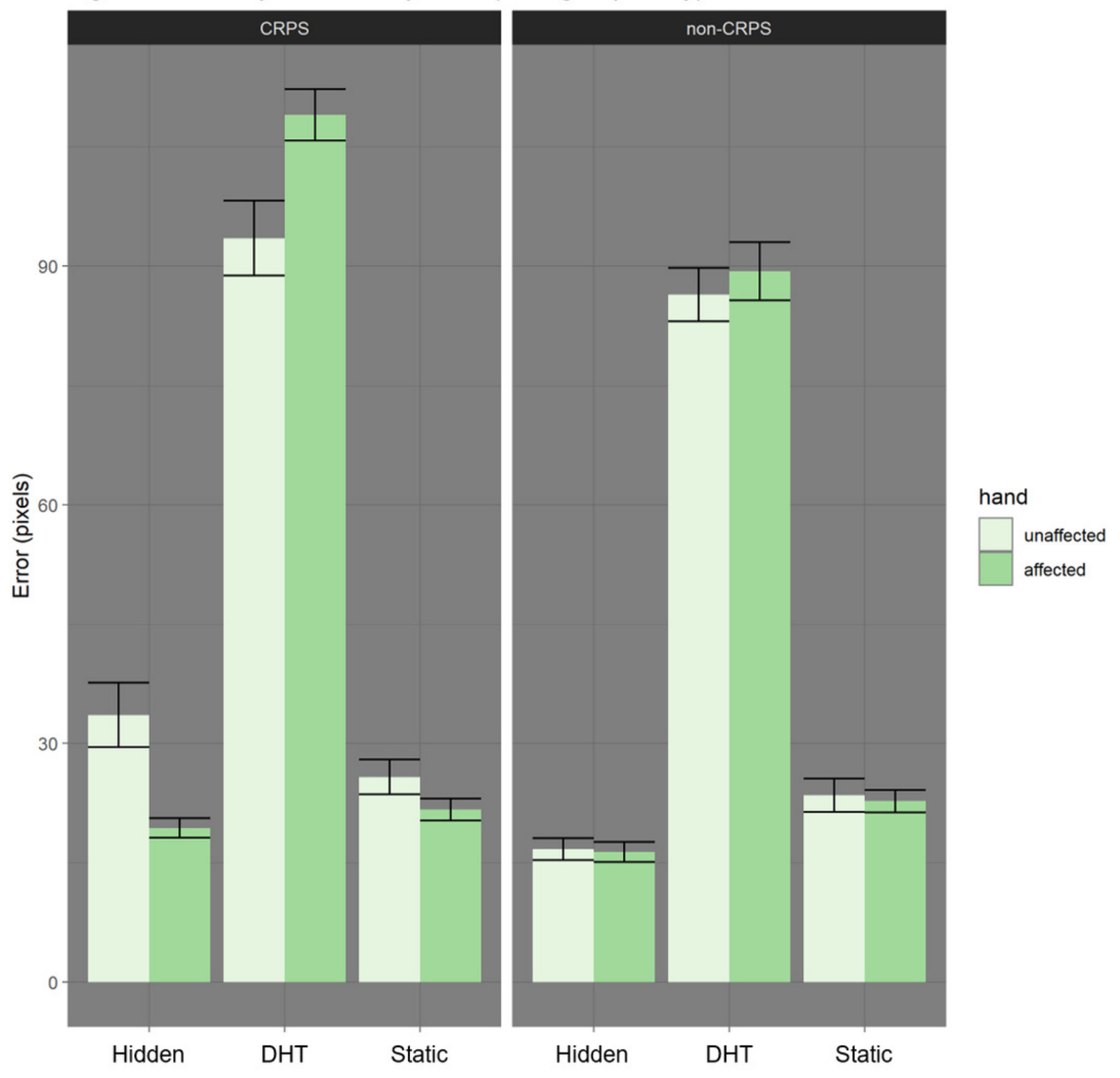




\section{Figure 4}

\section{Error by Age}

Fig. 4 - (a) Older age seems to be correlated with smaller error but only for the DHT condition, while the opposite trend can be observed for the other two conditions. This appears particularly clear when the two groups are considered separately (b), especially as far as the non-CRPS Pain group is concerned. However, panel (b) also shows that, in general, non-CRPS Pain group participants were older then the participants in the CRPS group. Interestingly enough, though, by comparing the affected and unaffected hand (c) (instead of the two groups) the effect is still present, but the difference between the DHT and the two control conditions is stronger for the affected compared to the unaffected hand. 
Figure 4a: Error by condition by age

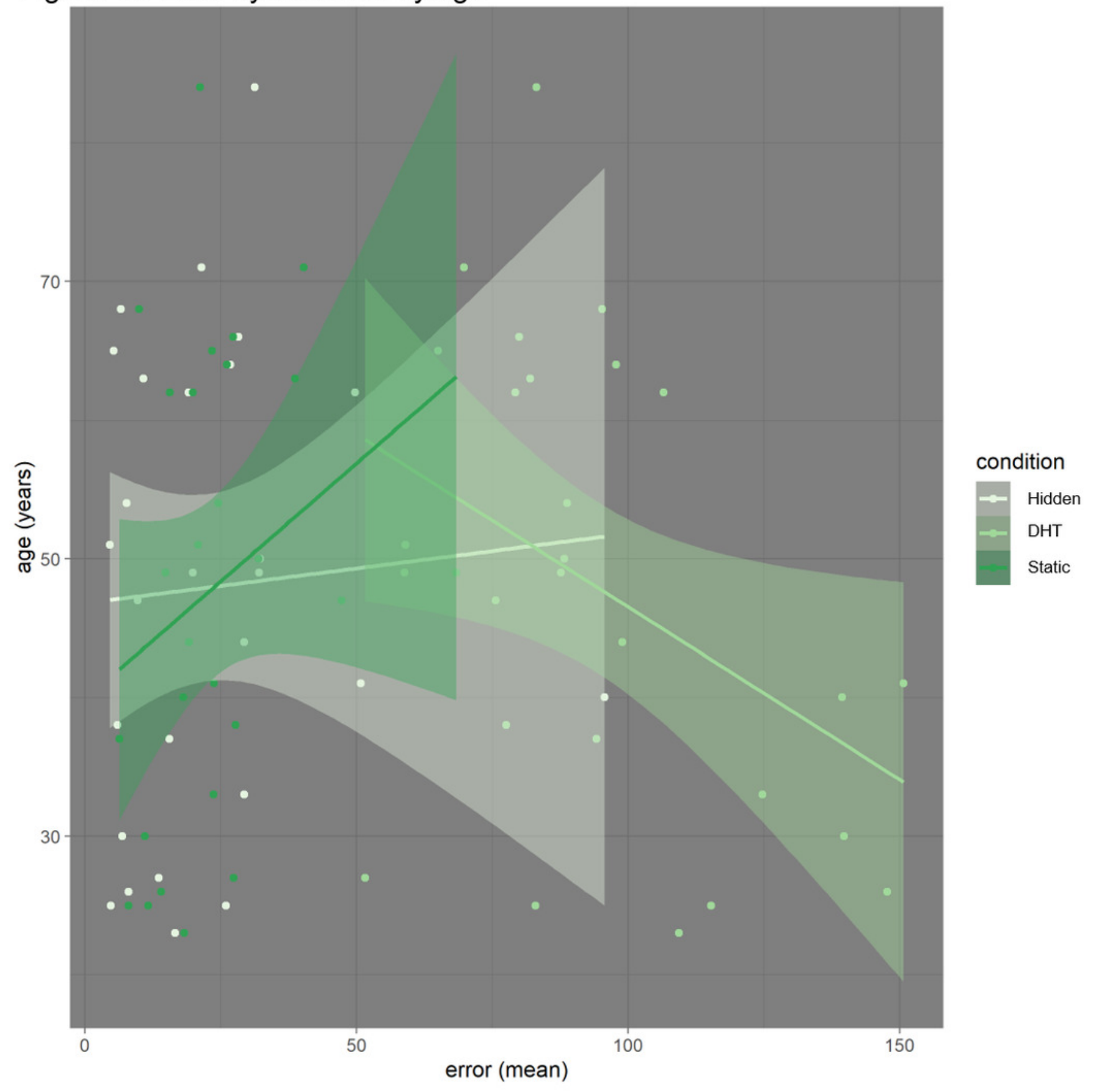


Figure 5

Error by age (groups)

Figure 4b: Error by condition by age (by group)

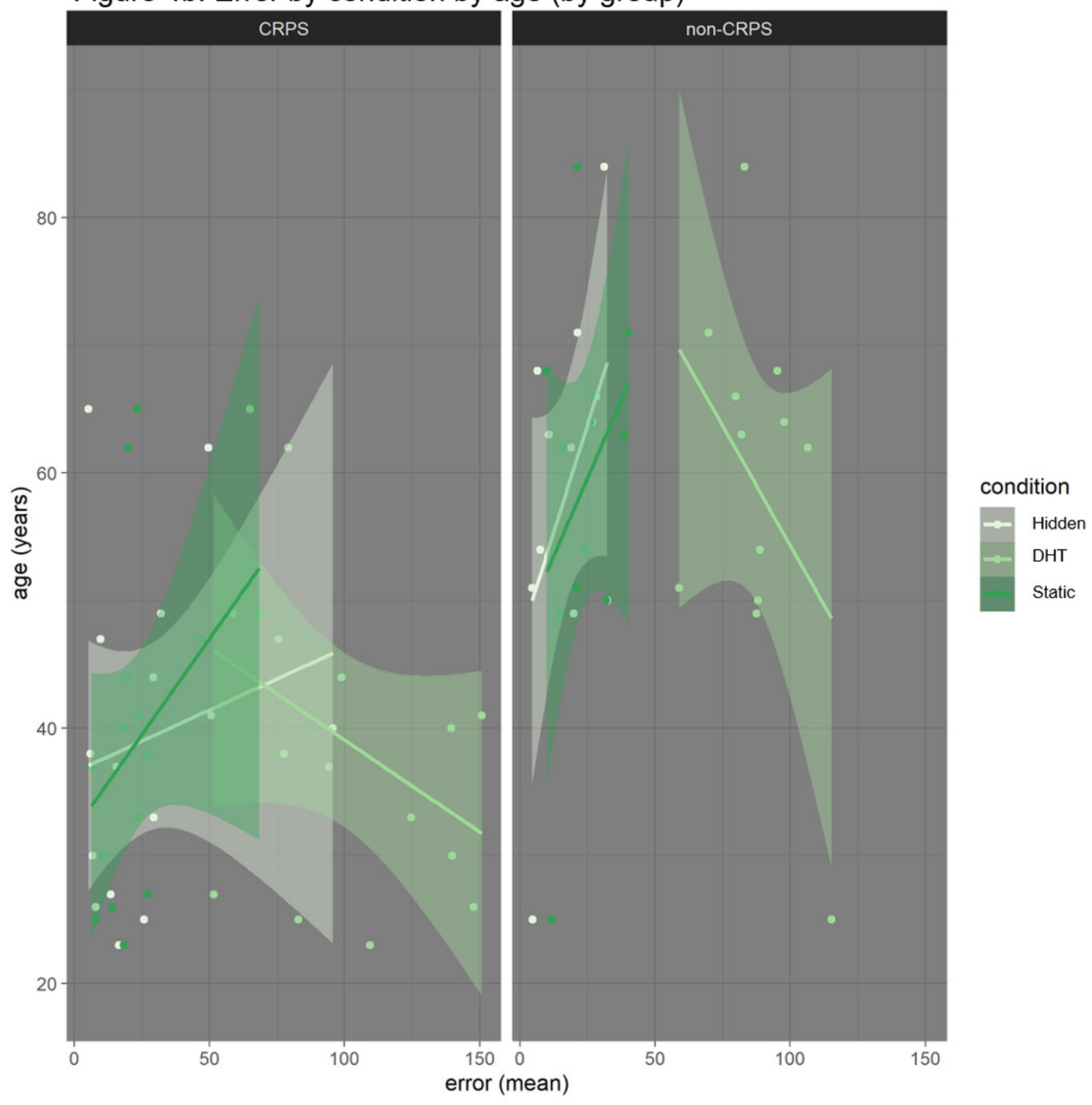


Figure 6

Error by age (Hand)

Figure 4c: Error by condition by age (by hand)

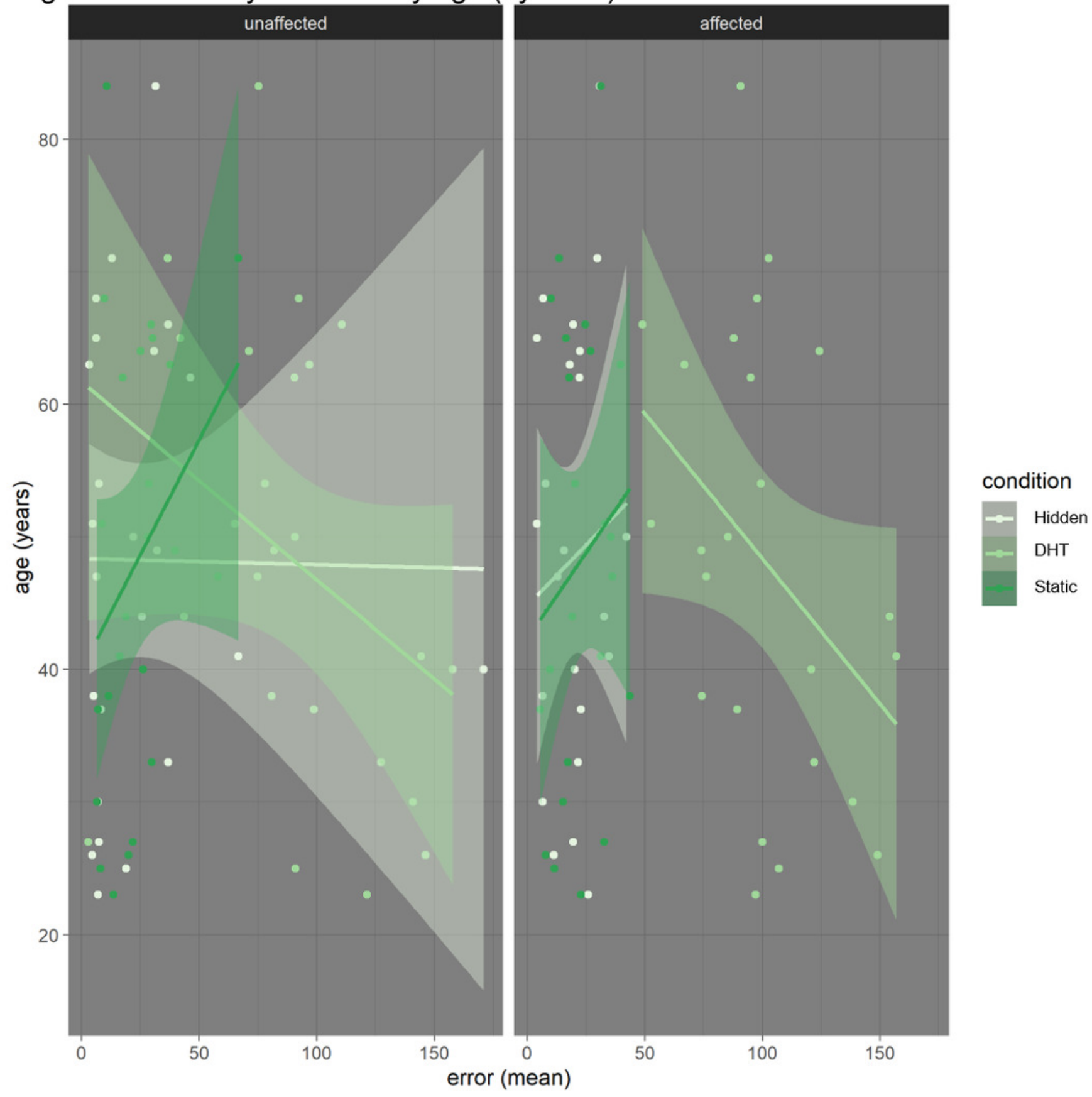




\section{Figure 7}

Anxiety by Error

Fig. 5 - The CRPS group (red line) shows the same positive correlation between anxiety and error across all conditions. The non-CRPS Pain (green line) and the Healthy (blue line) groups show the same positive correlation but only for the DHT condition (and the opposite for the control conditions). 
Figure 5: Effect of anxiety on error

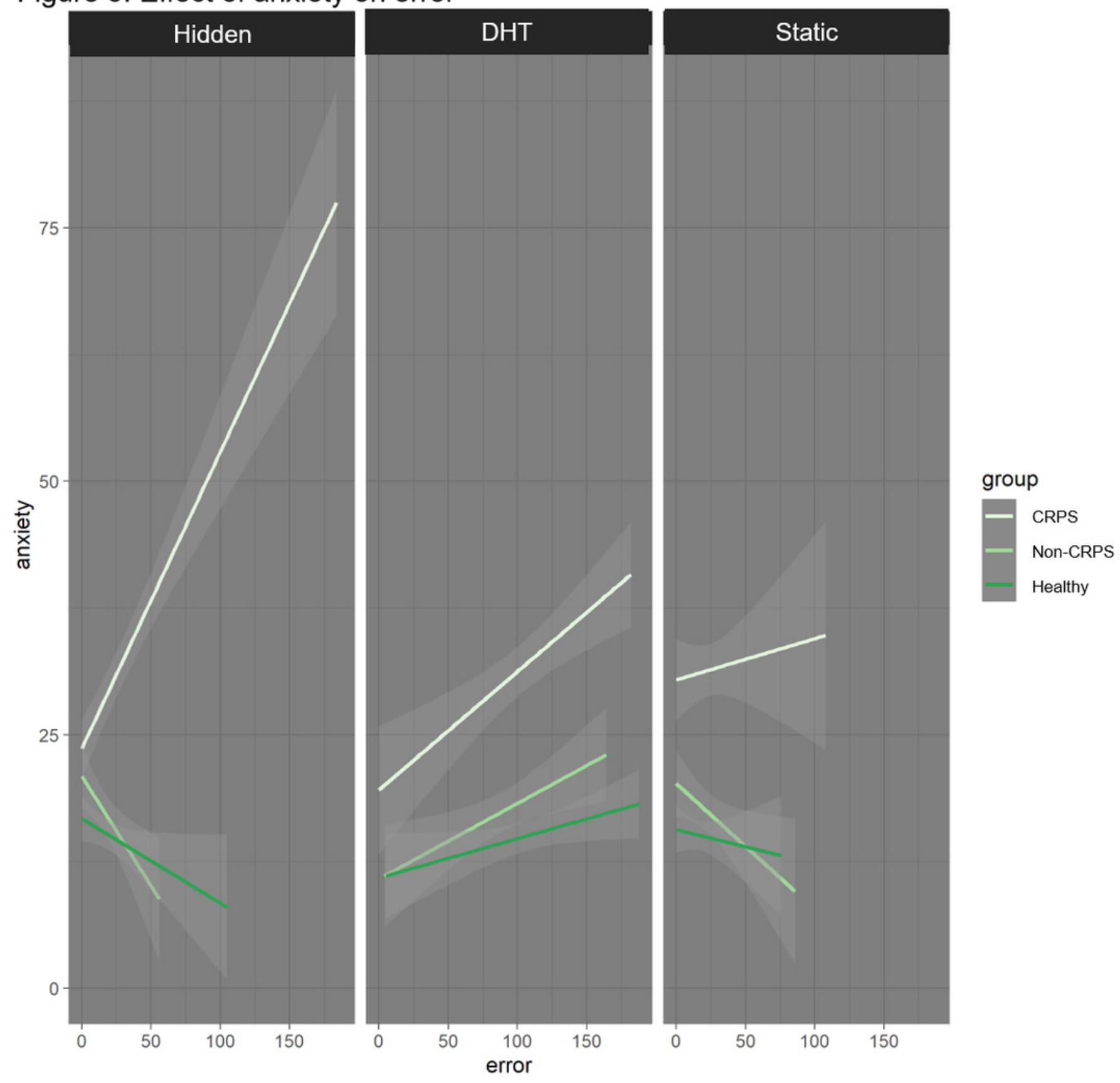


Table $\mathbf{1}$ (on next page)

Inclusion criteria 
1 Table 1:

2 Inclusion criteria

3

\begin{tabular}{|c|c|}
\hline & Inclusion Criteria \\
\hline General (All Groups) & $\begin{array}{l}\text { 1. Being over 18; } \\
\text { 2. Ability to read and understand spoken or written English; } \\
\text { 3. Absence of dermatological Condition on the upper limbs (that might } \\
\text { disrupt peripheral sensations); } \\
\text { 4. Normal or corrected to normal vision; } \\
\text { 5. No known psychiatric disorder }{ }^{1}\end{array}$ \\
\hline Pain-Free Control Group & $\begin{array}{l}\text { 1. Pain free and normal arms and Hands sensations; } \\
\text { 2. No past story or current neurological issues (e.g. traumatic brain } \\
\text { injuries, stroke, nerve injuries) or other medical Conditions; }\end{array}$ \\
\hline CRPS Group & $\begin{array}{l}\text { 1. Diagnosis of upper limb } \text { CRPS }^{2} \text {; } \\
\text { 2. No unresolved neurological or orthopaedic injury; } \\
\text { 3. Pain elsewhere. }\end{array}$ \\
\hline Non-CRPS Group & $\begin{array}{l}\text { 1. Non-CRPS type persistent (lasting }>3 \text { months) upper limb pain; } \\
\text { 2. No unresolved neurological or orthopaedic injury; } \\
\text { 3. Pain elsewhere. }\end{array}$ \\
\hline
\end{tabular}


Table 2 (on next page)

Localisation error: CRPS and Non-CRPS Pain groups

Localisation error (in pixels) for the two pain groups across the three different conditions. 
1 Table 2

\begin{tabular}{|l|l|l|}
\cline { 2 - 3 } \multicolumn{1}{c|}{} & \multicolumn{2}{c|}{ Pain groups (M \pm SD) (px) } \\
\cline { 2 - 3 } \multicolumn{1}{c|}{} & CRPS & Non-CRPS \\
\hline DHT & $101.26 \pm 43.29$ & $87.9 \pm 34.84$ \\
\hline Hidden & $26.48 \pm 32.28$ & $16.56 \pm 13.48$ \\
\hline Static & $23.74 \pm 19.65$ & $23.12 \pm 18.09$ \\
\hline
\end{tabular}

2

3

4 


\section{Table 3(on next page)}

Localisation error: Affected and Unaffected hand

Localisation error (in pixels) for the two pain groups across the three different conditions comparing the affected and the unaffected hand. 
1 Table 3

\begin{tabular}{|l|l|l|}
\cline { 2 - 3 } \multicolumn{1}{c|}{} & \multicolumn{2}{c|}{ Hand (M \pm SD) (px) } \\
\cline { 2 - 3 } \multicolumn{1}{c|}{} & Affected & Unaffected \\
\hline DHT & $99.51 \pm 37.02$ & $90.09 \pm 43.05$ \\
\hline Hidden & $17.93 \pm 13.04$ & $25.44 \pm 33.21$ \\
\hline Static & $22.21 \pm 14.56$ & $24.68 \pm 22.37$ \\
\hline
\end{tabular}

2

3 